\title{
Comparisons of Ice Cloud Parameters Obtained by Combined Remote Sensor Retrievals and Direct Methods
}

\author{
Sergey Y. Matrosov \\ Cooperative Institute for Research in Environmental Sciences, University of Colorado/NOAA, Boulder, Colorado
}

ANDREW J. HeYMSFIELD

National Center for Atmospheric Research, Boulder, Colorado

Robert A. Kropfli, Brooks E. Martner, and Roger F. Reinking

NOAA/Environmental Technology Laboratory, Boulder, Colorado

JACK B. SNIDER

Cooperative Institute for Research in Environmental Sciences, University of Colorado/NOAA, Boulder, Colorado

Paivi Pitronen and Edwin W. Eloranta

University of Wisconsin-Madison, Madison, Wisconsin

(Manuscript received 27 November 1996, in final form 16 May 1997)

\section{ABSTRACT}

\begin{abstract}
Ice cloud microphysical parameters derived from a remote sensing method that uses ground-based measurements from the Environmental Technology Laboratory's $\mathrm{K}_{\mathrm{a}}$-band radar and an IR radiometer are compared to those obtained from aircraft sampling for the cirrus priority event from the FIRE-II experiment. Aircraft cloud samples were taken not only by traditional two-dimensional probes but also by using a new video sampler to account for small particles. The cloud parameter comparisons were made for time intervals when aircraft were passing approximately above ground-based instruments that were pointed vertically. Comparing characteristic particle sizes expressed in terms of median mass diameters of equal-volume spheres yielded a relative standard deviation of about $30 \%$. The corresponding standard deviation for the cloud ice water content comparisons was about 55\%. Such an agreement is considered good given uncertainties of both direct and remote approaches and several orders of magnitude in natural variability of ice cloud parameters. Values of reflectivity measured by the radar and calculated from aircraft samples also showed a reasonable agreement; however, calculated reflectivities averaged approximately $2 \mathrm{~dB}$ smaller than those measured. The possible reasons for this small bias are discussed. Ground-based and aircraft-derived particle characteristic sizes are compared to those available from published satellite measurements of this parameter for the cirrus priority case from FIRE-II. Finally, simultaneous and collocated, ground-based measurements of visible $(0.523 \mathrm{~nm})$ and longwave IR $(10-11.4 \mu \mathrm{m})$ ice cloud extinction optical thickness obtained during the 1995 Arizona Program are also compared. These comparisons, performed for different cloud conditions, revealed a relative standard deviation of less than 20\%; however, no systematic excess of visible extinction over IR extinction was observed in the considered experimental events.
\end{abstract}

\section{Introduction}

Clouds are recognized as one of the main components of the earth's climate system. They influence shortwave solar radiation and infrared (IR) terrestrial radiation to modify the radiation budget of the atmosphere and the

Corresponding author address: Dr. Sergey Y. Matrosov, NOAA/ ERL/ETL, R/E/ET6, 325 Broadway, Boulder, CO 80303.

E-mail: smatrosov@etl.noaa.gov underlying surface. Quantitatively, this influence depends on the cloud macrophysical (e.g., cloud horizontal and vertical extent, persistence) and microphysical (e.g., cloud phase, characteristic cloud particle size, particle shape, liquid or ice water content) parameters that determine cloud radiative properties. Uncertainties still remain in relations between cloud radiative and microphysical properties (e.g., the excess cloud absorption anomaly) and also in statistics of cloud microphysics.

Several field programs have been conducted recently to resolve some of these uncertainties. Some of the ma- 
jor projects are the Atlantic Stratocumulus Transition Experiment (ASTEX) devoted mostly to liquid water clouds (Randall 1995) and the First International Satellite Cloud Climatology Project (ISCCP) Regional Experiment, Phases I and II (FIRE-I and -II), where highaltitude ice clouds such as cirrus were the prime object of studies (Starr and Wylie 1990; Stephens 1995). Many remote and direct measurements of cloud geometrical, radiative, and microphysical properties were conducted from different observational platforms (e.g., satellite, aircraft, balloon, ground) during these projects.

Cloud data collected primarily by traditional aircraft sampling techniques have been the main source of our knowledge about cloud microphysics. Now a number of remote sensing methods to derive cloud microphysical and radiative parameters over extended periods of time are becoming available. These methods are usually satellite- or ground-based and use active (radars, lidars) and/or passive (radiometers) remote sensors operating at different wavelengths. The great advantage of such methods is their ability to provide a much greater spatial and temporal coverage compared to traditional aircraft sampling.

The problem of relating cloud properties obtained by remote and direct methods remains very important. Sometimes remote methods produce results that are quite different from those obtained by aircraft sampling. An example of such a discrepancy, which is not very well understood, is the occasional differences in cirrus cloud effective particle sizes inferred from satellite radiometric measurements and aircraft sampling (Sassen 1990). While some deficiencies of traditional two-dimensional (2D) particle probes are realized (e.g., unsatisfactory sampling of small particles), accuracies of remote sensing methods have not been well studied and understood until now.

It is possible to estimate these accuracies theoretically making reasonable assumptions about measurement errors and uncertainties of a priori information and models. However, in a way, a more robust way to understand and assess these accuracies is to compare the simultaneous results of a remote method with direct sampling or the results of two or more different remote methods for the same or at least close cloud volumes. Such comparisons are difficult because of different sampling volumes for different techniques. Nevertheless, they are a necessary step in validation of the remote sensing methods.

This paper presents comparisons of such ice cloud microphysical parameters as particle characteristic size and ice water content (IWC) obtained with the groundbased radar-IR radiometer method during FIRE-II (Matrosov 1997) with in situ measurements of these parameters from research aircraft. Radar-radiometer-derived particle sizes are also compared to available satellitederived values of this parameter.

The cloud IR absorption optical thickness $\tau_{a}$, being a by-product of the radar-radiometer method, is also an important radiative cloud parameter. Extinction optical thickness $\tau_{e}$ is estimated from $\tau_{a}$ and particle characteristic size. Results of $\tau_{e}$ estimations are compared with visible optical thickness data available from simultaneous and collocated lidar measurements during the 1995 Arizona Program field experiment (Klimowski 1995).

\section{Remote and in situ approaches to collect microphysical cloud data}

Comparisons of remotely obtained cloud microphysical parameters with in situ direct measurements from aircraft are always difficult to perform because of the large disparity in sample volumes. Aircraft usually collect data when flying along a horizontal leg at speeds of about 100-200 $\mathrm{m} \mathrm{s}^{-1}$. The usual time interval of 5 s necessary to get reasonable sample statistics provides an average horizontal resolution of the order of $500 \mathrm{~m}$ to $1 \mathrm{~km}$. Furthermore, estimations of vertical profiles of cloud microphysical parameters from aircraft measurements are hampered by sampling a cloud at various altitudes at different times during which the cloud may have evolved. Balloonborne measurements provide vertical profiles of cloud microphysics only in the Langrangian sense, while models usually require the $\mathrm{Eu}-$ lerian types of vertical profiles.

In this section, comparisons are given for cirrus cloud characteristic particle sizes and cloud IWC (sometimes this parameter is called ice mass content or IMC). The remote sensing technique used here was the radar-radiometer method previously described by Matrosov et al. (1994). The in situ cloud sampling was performed from the King Air and Sabreliner research airplanes operated by the National Center for Atmospheric Research (NCAR). Note that some comparisons of the same kind for particle sizes were given by Matrosov et al. (1995). However, only King Air data, which did not account adequately for the smallest cloud particles, were included in those comparisons. Here, we extend comparisons also to cloud IWC and consider the Sabreliner data that have a much better measurement of small particles due to a special video sampling approach. Note that we define small particles here as those that are not measured by traditional $2 \mathrm{D}$ probes. The critical particle size below which particles do not trigger the probe, however, depends on the aircraft speed. So, according to this definition, small particle coverage is different for the King Air (100 $\mathrm{m} \mathrm{s}^{-1}$ flight speed) and the Sabreliner (175 $\mathrm{m} \mathrm{s}^{-1}$ flight speed).

\section{a. Brief description of the remote sensing method}

The remote sensing method uses combined measurements taken by a vertically pointed $\mathrm{K}_{\mathrm{a}}$-band $\operatorname{radar}(\lambda=$ $0.86 \mathrm{~cm})$ and a narrowband $(\lambda \sim 10-11.4 \mu \mathrm{m})$ IR radiometer. Radar measurables are vertical profiles of radar reflectivity $Z_{e}$ and Doppler velocity $V_{D}$, which is 
used to estimate cloud particle reflectivity-weighted fall velocities $V_{t}$. Brightness temperature measured by the IR radiometer is used to obtain $\tau_{a}$. The atmospheric temperature profile and water vapor amount, which are needed for this, are known from radiosonde soundings and data from a two-channel microwave radiometer $(21.6$ and $31.65 \mathrm{GHz})$, which provides a measure of this amount continuously. Radiometer measurements also provide information about cloud-integrated liquid water. Retrievals are performed only for pure ice phase clouds.

The remotely derived vertical profiles of cloud particle characteristic size $D_{m}$ (expressed in median mass diameters of equal-volume spheres) and their concentration $C$ are found as a result of an iterative procedure when the nonlinear system of algebraic equations is solved for each beam of radar data consisting of $N$ radar range samples with equal spacing $\Delta h_{i}=37.5 \mathrm{~m}(i=$ $1, \ldots, N)$ :

$$
\begin{aligned}
\tau_{a} & =\sum_{i} k_{1}\left(\rho, r, n, D_{m i}\right) C_{i} D_{m i}^{2} \Delta h_{i}, \\
Z_{e i} & =k_{2}\left(\rho, r, n, D_{m i}\right) C_{i} D_{m i}^{6},
\end{aligned}
$$

and

$$
V_{t i}=A k_{3}\left(\rho, r, n, D_{m i}\right) D_{m i}^{B} .
$$

In these equations coefficients $k_{1}, k_{2}$, and $k_{3}$ depend on a priori assumptions about particle density $\rho$ (usually $\rho$ is size dependent), particle aspect ratio $r$ (particles are modeled as plates or columns or the mix of these two habits), and the order of the particle size distribution $n$ (the shape of the distribution is approximated by gamma functions of different orders). Values of the exponent $B$ in the particle size-fall velocity relationship (3) vary slightly for typical falling regimes of cirrus particles (Mitchell 1996), and these values are also included in a priori assumptions. Values of the coefficient $A$, however, show a greater natural variability. As cloud microphysical parameters, the values of $A$ are found as a result of the iterative solution of the equation system (1)-(3) for each radar beam of data. The iterative scheme and estimated uncertainties of the cloud microphysical parameter retrievals are described in more detail in recent papers (Matrosov et al. 1995; Matrosov 1997).

\section{b. In situ aircraft sampling}

The NCAR King Air and Sabreliner research aircraft participated in the FIRE-II field experiment. Both aircraft were equipped with two Particle Measuring Systems Inc. 2D probes to detect and measure ice cloud particle distributions. The King Air 2D-C probe sized particles in 32 bins with maximum dimensions from about $25 \mu \mathrm{m}$ to $1 \mathrm{~mm}$ in $25-\mu \mathrm{m}$ increments. Zero area image particles with sizes approximately between 18 and $25 \mu \mathrm{m}$ still triggered the probe and thus were accounted for in total spectra. Hence, particles with the sizes less than about $18 \mu \mathrm{m}$ were small according to the definition given above for the King Air sampling and were not accounted for.

The King Air 2D-P probe sampled particles from about $50 \mu \mathrm{m}$ to above $3.2 \mathrm{~mm}$ in $100-\mu \mathrm{m}$ increments. The particle habits and size spectra were derived from composite 2D-C and 2D-P data. For each of the size spectra generated by averaging data over 5-s intervals, median mass size, cloud IWC, and radar reflectivity were derived among a number of other cloud parameters.

Due to higher aircraft speed, the Sabreliner 2D-C probe sampled cloud particles starting from above approximately $44 \mu \mathrm{m}$ (the resolution was $25 \mu \mathrm{m}$ along the probe array axis and $44 \mu \mathrm{m}$ in the direction of flight). The sizing interval of the Sabreliner 2D-C probe was $44 \mu \mathrm{m}$. The definition of small particles adopted here gives the critical size value of about $25 \mu \mathrm{m}$ because particles with cross-sectional areas of more than half of the resolution element $(25 \mu \mathrm{m}$ by $44 \mu \mathrm{m})$ still trigger the probe. During FIRE-II, the Sabreliner was sampling clouds at high altitudes, where particles' sizes did not exceed $1 \mathrm{~mm}$.

In addition to the $2 \mathrm{D}$ probes, the NCAR Sabreliner was equipped with a video ice particle sampler (VIPS) for measuring small ice particles. This instrument collects particles starting from sizes as small as about 5 $\mu \mathrm{m}$ on a continuous oil-coated belt. The particle images are then recorded with a video camera, digitized, and analyzed on a computer. This process is rather tedious and only a relatively small subset of the available data has been analyzed. The details of the VIPS and the data analyzing technique are given by McFarquhar and Heymsfield (1996). The combined VIPS/2D-C particle size spectra accounted for particles from about $5 \mu \mathrm{m}$ to about $1.5 \mathrm{~mm}$, which represents nearly the whole dynamic range of particle sizes in natural cirrus clouds. These composite spectra are probably one of the most complete data sources now available from aircraft sampling.

The aircraft sampled clouds at different locations. They flew stepped "racetracks" of different lengths at constant altitudes and also made spiral descents. The FIRE-II 26 November 1991 cirrus priority event provided a dataset when the ETL $\mathrm{K}_{\mathrm{a}}$-band radar and IR radiometer were operating at the Coffeyville, Kansas, experiment hub site simultaneously with the aircraft sampling cloud above the vicinity of the hub.

Both in situ and remote data show high vertical and horizontal variability of cirrus cloud parameters. Hence, for quantitative comparisons, only data collected when the aircraft was passing approximately above the ground-based remote sensors should be chosen. Examining coordinates of aircraft positions relative to the instrumentation hub reveals that aircraft were not passing directly overhead for the whole duration of the event (more than $3 \mathrm{~h}$ ). However, during this period, the King Air passed 10 times within $3 \mathrm{~km}$ from the point above 
the ground-based instruments when they were operating. The Sabreliner made five close proximity passes. The data collected during these King Air and Sabreliner passes were chosen for the quantitative comparisons.

\section{Comparisons of ice cloud parameters in FIRE-II obtained from aircraft and ground-based remote measurements}

\section{a. Comparisons of reflectivities measured by radar and inferred from aircraft samples}

One of the products that is calculated from in situ derived particle distributions is radar reflectivity factor $Z$. Values of $Z$ calculated from particle samples are sometimes used to derive empirical relationships between cloud parameters and radar reflectivity (e.g., Brown et al. 1995; Sassen 1987; Liao and Sassen 1994). Such relationships are then used in some remote sensing applications, so it is important to verify such procedures for calculating radar reflectivity from in situ samples against the real simultaneous radar measurements.

The procedure of calculating reflectivity of ice particle distributions from in situ aircraft sampling can be briefly described as follows. Sampled particles are classified by shape (habits). Using a pattern recognition algorithm, the habit of each particle is classified and size spectra are derived for each particle type. Then the habit-dependent mass-length ( $m$ and $L$, respectively) relationships are used to calculate melted diameter $D_{\text {mel }}$ for particles of each size and habit:

$$
m_{i j}=e L_{i j}^{f}=(\pi / 6) D_{\text {mel }}^{3},
$$

where $e$ and $f$ are habit- and size-dependent. The summation of $D_{\text {mel }}^{6}$ is then performed with respect to size classes $i$. In each size class, another summation is also done accounting for fractions of particles with different habits $j$. A 7-dB factor is then subtracted from the result (expressed in $\mathrm{dBZ}$ ) to obtain water equivalent radar reflectivity $Z_{e}$.

Note that if the mass of a particle is preserved (as is done in 2D analysis when calculating the melted diameter using mass-length relationships), reflectivity of such a particle practically does not depend on its density $\rho$ because $K / \rho \approx$ const [here, $K=\left|\left(m^{2}-1\right) /\left(m^{2}+2\right)\right|$, $m$ is the refractive index]. So the $7-\mathrm{dB}$ correction is valid for particles with different densities. Of course, particles with the same size and different densities (which would result in different mass) have different reflectivities. In more detail, the procedure of calculating $Z_{e}$ from the result of aircraft sampling is described by Heymsfield (1977). That paper also gives values of coefficients $e$ and $f$ for different particle habits, which are used here.

Note that the described scheme of calculating $Z_{e}$ assumes the Rayleigh type of scattering and neglects effects of particle nonsphericity. Non-Rayleigh scattering effects in cirrus clouds are usually very small for radar frequencies at $\mathrm{K}_{\mathrm{a}}$ band and lower (Matrosov 1993). Reflectivities of nonspherical particles are often larger than those of equal-volume spheres of the same density (Atlas et al. 1953), so this calculation method could somewhat underestimate $Z_{e}$.

Figure 1 shows comparisons of reflectivity values measured by the NOAA $\mathrm{K}_{\mathrm{a}}$-band radar with those calculated using the method described above. Aircraft altitudes (above the ground) during the passes approximately above the ground-based remote sensors and the time of these passes are also shown in this figure. Square and circle symbols show comparisons for the King Air and Sabreliner data, respectively.

There is a general agreement between measured and calculated reflectivities. Measured values of $Z_{e}$ are somewhat higher than those calculated from particle samples. However, most of the differences do not exceed about $2 \mathrm{~dB}$, which is about the order of uncertainty of reflectivity measurements. The small bias of the measured $Z_{e}$ values being a little larger can be, in part, explained by possible particle nonsphericity effects on radar reflectivity, uncertainties of $Z_{e}$ calculations from particle spectra, radar calibration errors, and sampling volume differences between the radar and aircraft.

To assess the uncertainty of the $Z_{e}$ calculations from in situ distributions, such calculations were also performed with $e$ and $f$ coefficients [Eq. (4)] given by Brown and Francis (1995) instead of original coefficients from Heymsfield (1977). The calculated $Z_{e}$ values in this case are slightly greater than those shown in Fig. 1 ; the average increase is about $1.5 \mathrm{~dB}$. This would make the agreement between calculated and measured reflectivities even better than shown in Fig. 1. We can conclude here that, given uncertainty of both measurements and calculations, the overall agreement between calculated and measured $Z_{e}$ is considered good.

\section{b. Comparisons of particle sizes retrieved remotely and measured by in situ sampling}

A standard data processing technique uses particle distributions inferred from in situ aircraft measurements to calculate a median mass size $L_{m}$ (expressed in terms of particle major dimensions in 2D images) for each sample. The radar-radiometer remote sensing method retrieves characteristic sizes in terms of median mass diameters of equal-volume spheres $D_{m}$. The following comparisons are given in terms of $D_{m}$. The relation between $L_{m}$ and $D_{m}$ was considered by Matrosov et al. (1995).

Figure 2 shows comparisons of characteristic particle sizes for the same aircraft passes above the groundbased instruments as in Fig. 1. When performing retrievals using the radar-radiometer method, the following assumptions were made: the cloud particles are a mix of plates and columns with aspect ratio 0.5 (the ratio $L_{m} / D_{m}$ is about 1.3 for this assumption), effective particle density decreases with size as $D^{-1.1}$ (for $D>$ 


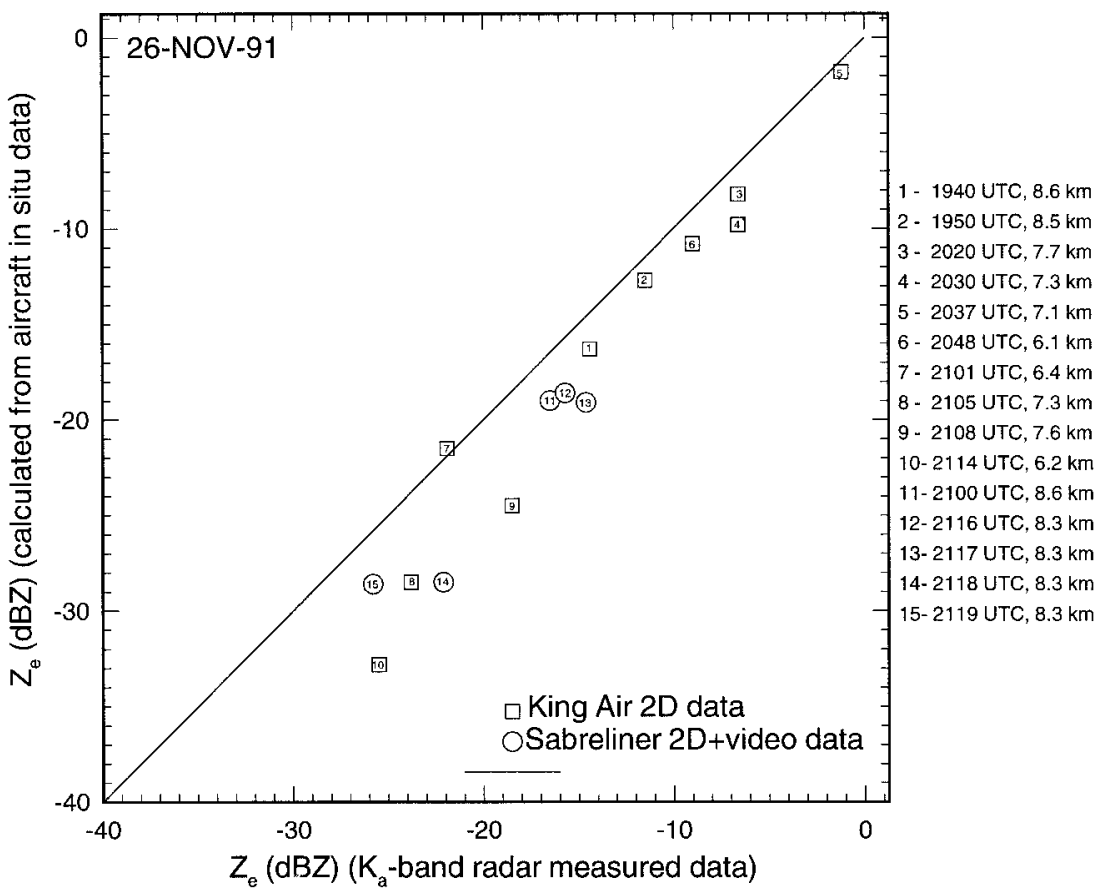

FIG. 1. Comparison of reflectivity values measured by ETL $\mathrm{K}_{\mathrm{a}}$-band radar and calculated from aircraft particle samples. Comparisons are given for time moments when aircraft was passing approximately above ground-based instruments.

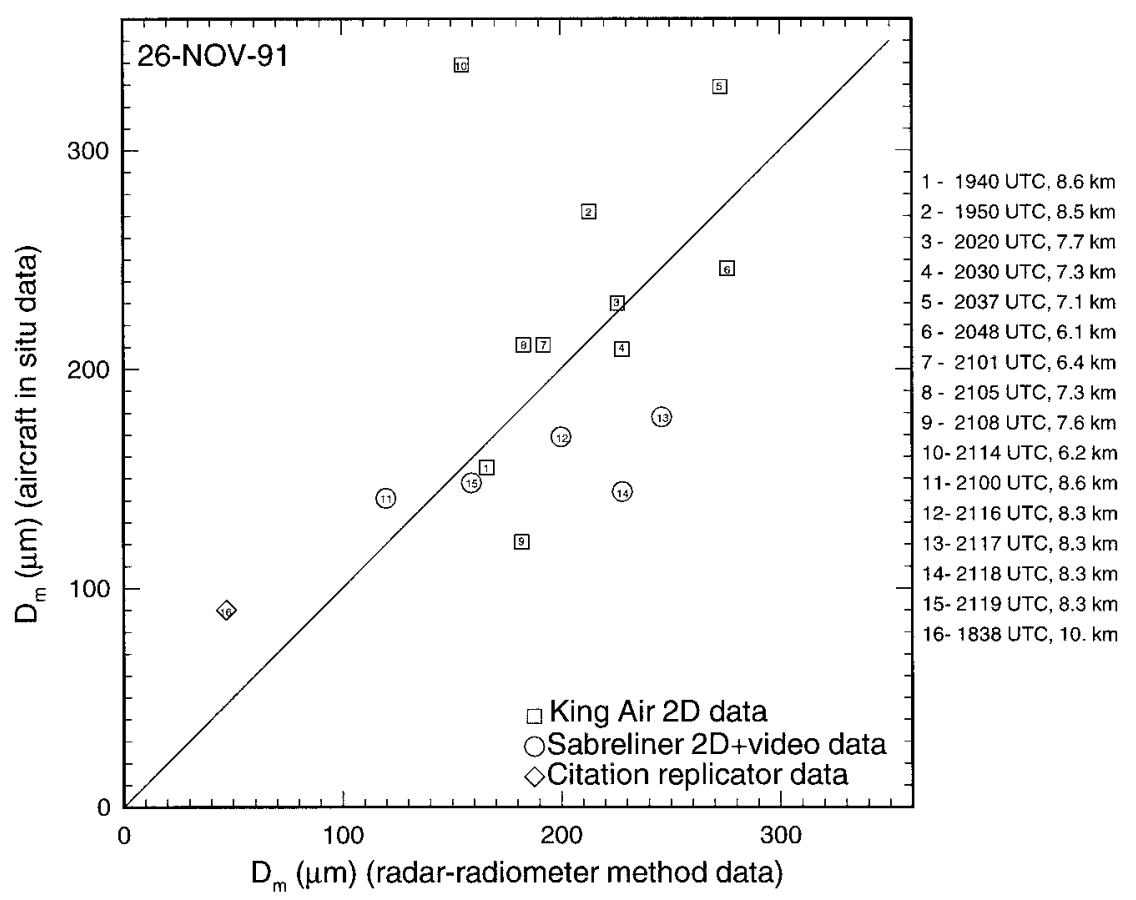

FIG. 2. Comparisons of cloud particle characteristic sizes expressed in terms of median mass diameters of equal-volume spheres as derived from remote radar-radiometer measurements and from aircraft sampling. Comparisons are given for time moments when aircraft was passing approximately above ground-based instruments. 


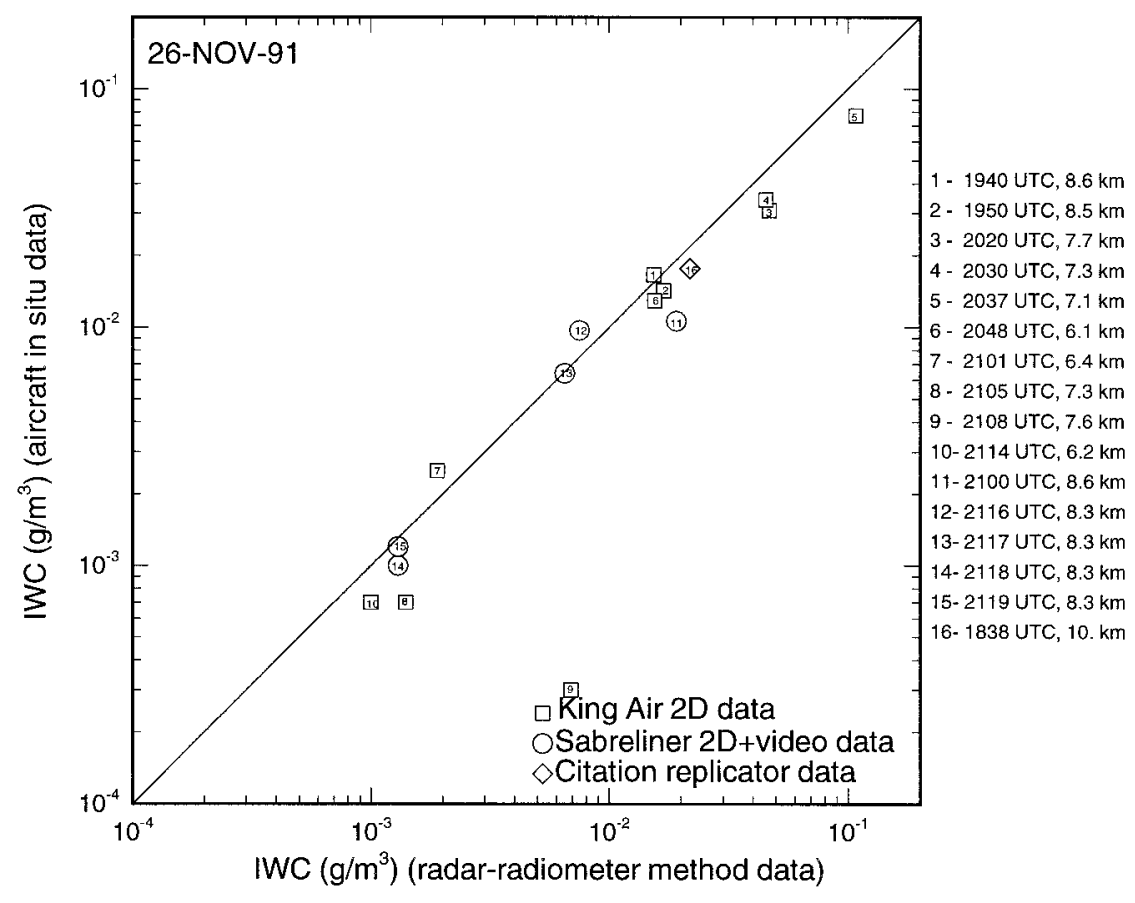

FIG. 3. Same as Fig. 2 but for the cloud ice water content.

$100 \mu \mathrm{m})$ as suggested by Brown and Francis (1995), the shape of the size distribution is approximated by the gamma function of the first order, and the exponent $B$ in (3) changes from about 0.9 for small particles $(D \sim$ $20 \mu \mathrm{m})$ to 1.1 for larger particles $(D>500 \mu \mathrm{m})$.

In addition to the King Air and Sabreliner data comparisons, Fig. 2 shows a comparison for the size measured by the Desert Research Institute formvar replicator available for this case (P. Arnott 1997, personal communication). The horizontal distance between the radar and the University of North Dakota Citation research aircraft with this replicator aboard was about $9 \mathrm{~km}$. This measurement was made near the cloud top. For estimating $D_{m}$ from the reported $L_{m}$, the same ratio (1.3) as for the King Air and Sabreliner data was used. The value of this ratio, however, could be somewhat too low because the particles sampled by the replicator were mostly elongated polycolumns, single plates, and needles.

The sensitivity of the retrieval results to these assumptions is discussed in detail by Matrosov et al. (1995). Note that changing the assumption about the larger particles' density by $20 \%$, or changing the order of the assumed gamma function size distribution to 0 or 2 (from 1), or changing the assumed particle aspect ratio from 0.5 to 1.0 (i.e., assuming the spherical particle shape) causes variations of the retrieved values of $D_{m}$ of not more than $10 \%$ for the typical particle sizes compared here.

The characteristic size such as $D_{m}$ describes the whole particle distribution, and individual particle sizes could vary as much as three orders of magnitude for a fixed value of $D_{m}$. The compared values of $D_{m}$, obtained using both the remote sensing method and in situ sampling, generally ranged from about $100 \mu \mathrm{m}$ to slightly above $300 \mu \mathrm{m}$.

For the data in Fig. 2, the relative standard deviation of particle median sizes calculated using in situ samples from those retrieved using remote sensor measurements is about $33 \%$. As it is evident from Fig. 2, the agreement is not that much different for comparisons of remotely measured median sizes with those from King Air 2D spectra and Sabreliner combined 2D and video spectra. This indicates that small particles $(D<25 \mu \mathrm{m})$, at least in the considered cloud samples, did not contribute much to estimates of median mass particle sizes.

\section{c. Comparisons of cloud ice water content retrieved remotely and measured by in situ sampling}

Compared to the characteristic size (not individual sizes) of particle distribution, the cloud IWC has a much greater dynamic range of natural variations. IWC in cirrus cloud can vary more than four orders of magnitude (Dowling and Radke 1990) from $10^{-4} \mathrm{~g} \mathrm{~m}^{-3}$ to $1.2 \mathrm{~g} \mathrm{~m}^{-3}$. Given such a great dynamic range, any remote sensing method able to provide IWC data even with an accuracy of factor of about 2 will be extremely useful for many applications ranging from climate modeling to cloud physics.

The comparisons of IWC values calculated from aircraft samples with those retrieved using the radar-radiometer method are presented in Fig. 3. Again, as in Fig. 1 and 2, the data are shown only for the aircraft 
passes approximately above the ground-based instruments. The variability range of IWC for those passes, from both direct and remote datasets, is more than two orders of magnitude: from less than $10^{-3} \mathrm{~g} \mathrm{~m}^{-3}$ to almost $0.1 \mathrm{~g} \mathrm{~m}^{-3}$.

As shown in Fig. 3 the agreement between IWC derived remotely and directly is surprisingly good (except for one comparison point at 2108 UTC) given the high dynamic range of IWC changes. The relative standard deviation of one dataset relative to the other is $55 \%$ for all the presented data. The standard deviation with the exclusion of the comparison point at 2108 UTC is less than $40 \%$.

Note that the quality of the agreement is approximately the same for IWC derived from King Air and Sabreliner samples. This again reflects the fact that smallest particles usually do not contribute much in the total cloud mass. It was mentioned, for example, in previous studies (Heymsfield et al. 1990) that particles with sizes $25-100 \mu \mathrm{m}$ usually contribute about $10 \%$ in total IWC, and particles smaller than $25 \mu \mathrm{m}$ are a negligible percentage of the total mass. A similar conclusion was also reached by Brown et al. (1995). For these comparison data, the contribution from the data from VIPS (i.e., from particles less than about $25 \mu \mathrm{m}$ ) was only about $3 \%-4 \%$ to the total IWC from the combined Sabreliner samples. It should be noted, however, that the contribution of small particles can be more significant in other cases, for example, in cold anvils and contrails (Heymsfield and McFarquhar 1996).

As for calculated $Z_{e}$, IWC values calculated from aircraft sampling are subject to variations due to different mass-size relationships (4) used in these calculations. This variability was assessed by using the relationships given by Brown and Francis (1995) and Mitchell (1996) instead of the Heymsfield (1977) relationships used to calculate data in Fig. 3. The use of the Brown and Francis relationship results in about a 30\%-35\% increase in the calculated IWC values, while the use of Mitchell's relationships for cirrus particles results in a decrease in calculated IWC by a similar amount.

The sensitivity of IWC values retrieved from radarradiometer measurements to a priori assumption uncertainties and measurement errors was discussed by Matrosov et al. (1994). Generally, uncertainties in the retrieved IWC due to assumptions about the order of the gamma distribution and exponent $B$ in (3) are about a factor of 2 greater than those for $D_{m}$ (see the retrieval uncertainties for $D_{m}$ in section $2 \mathrm{~b}$ ). The sensitivity to the density assumption is also somewhat greater for IWC than for $D_{m}$. However, IWC is less sensitive than $D_{m}$ to the assumption about particle aspect ratio. Generally, combined retrieval uncertainties of IWC are greater than those for the median mass size by $50 \%-80 \%$.

Taking into consideration the great dynamic range of IWC changes and uncertainties of both remote and direct measurement approaches, we conclude that the agree- ment between data these approaches provided is quite satisfactory.

\section{Comparisons of particle sizes obtained by the radar-radiometer method and aircraft sampling with the satellite data}

The multiagency FIRE-II field experiment also included satellite measurements of cirrus clouds. Some satellite data are available from published sources (e.g., Ou et al. 1995). Here, we also compare cloud parameters derived with the radar-radiometer method (groundbased remote data) with available satellite-derived data. This comparison is given for the same cirrus event for which remote and aircraft data were compared.

The satellite retrieval scheme (Ou et al. 1995) uses upwelling IR radiances from the Advanced Very High Resolution Radiometer (AVHRR) flown on the NOAA11 satellite. The radiance data from 3.7- and $10.9-\mu \mathrm{m}$ AVHHR channels are used to retrieve cloud optical thickness and particle characteristic size. The NOAA-11 satellite passed the Coffeyville hub only twice a day with a viewing geometry favorable for retrievals. One of the passes occurred at about 2111 UTC 26 November 1991 when the ground-based instrumentation was operating. Aircraft data above the hub were also available for times close to 2111 UTC.

The size information retrieved from satellite measurements is given in terms of effective size $D_{e}$. Unlike the radar-radiometer method, the passive satellite measurements do not provide vertically resolved information. So $D_{e}$ is the size averaged throughout the cloud layer, which at 2111 UTC was about $3.5 \mathrm{~km}$ thick. The satellite retrievals of $\mathrm{Ou}$ et al. (1995) show that values of $D_{e}$ about $60 \mu \mathrm{m}$ are present around Coffeyville at 2111 UTC. However, according to the satellite data map, the Coffeyville hub was located within a region of high horizontal gradient of $D_{e}$.

To compare size information from different sources, it should be expressed in the same terms as was done for comparisons shown in Fig. 2. According to an empirical relationship provided by $\mathrm{Ou}$ et al. (1995), the satellite-derived value of $D_{e} \approx 60 \mu \mathrm{m}$ corresponds to the effective radius of an equivalent area sphere, $r_{\text {ea }} \approx$ $50 \mu \mathrm{m}$. As was mentioned before, the radar-radiometer method expresses results in median mass diameters of equal-volume spheres $D_{m}$.

For size distributions described by gamma functions of small orders [according to Kosarev and Mazin (1989), these functions, in most cases, satisfactorily describe experimental cirrus size spectra], $D_{m} \approx 2.4 r_{\mathrm{ev}}$, where $r_{\mathrm{ev}}$ is the effective radius of a equal-volume sphere. Note that a similar relation was also reported by Heymsfield and McFarquhar (1996) from experimental spectra. The relation between $r_{\text {ea }}$ and $r_{\mathrm{ev}}$ depends on particle shape and orientation. The difference between these two sizes, however, should not be very significant for cirrus particles with moderate aspect ratios, and we will ignore 


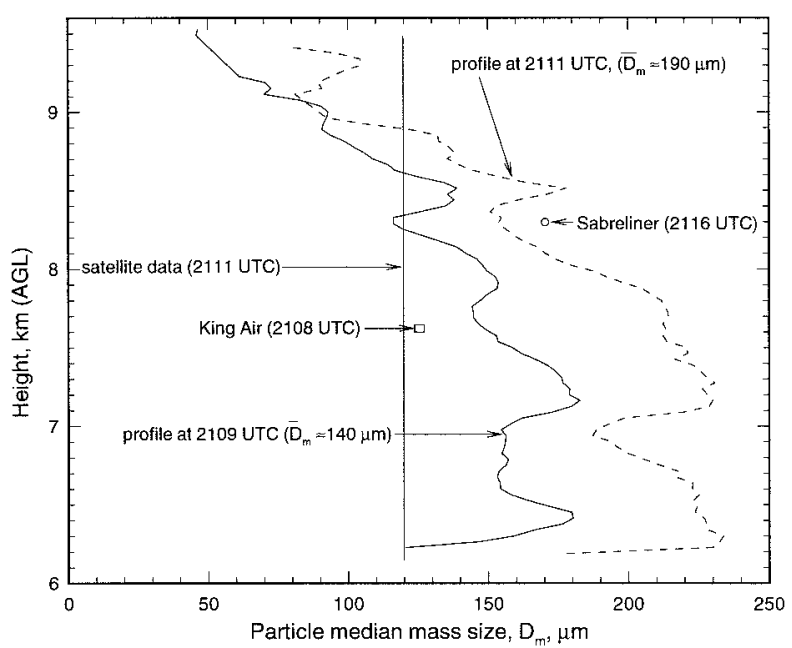

FIG. 4. Profiles of cloud particle characteristic sizes from radarradiometer ground-based measurements and available aircraft size estimations at corresponding heights for times in the nearest proximity of the satellite estimation of the mean cloud particle size (about 120 $\mu \mathrm{m}$ as expressed in terms of median mass diameters of equal-volume spheres).

it for this comparison. Summarizing, we conclude that satellite measurements yielded $\bar{D}_{m} \approx 120 \mu \mathrm{m}$ near Coffeyville at about 2111 UTC. The overbar here means that this is a $D_{m}$ value averaged throughout cloud vertical extent.

Figure 4 shows two vertical profiles of $D_{m}$ retrieved using the radar-radiometer method for measurements 2 min apart. As was mentioned above, satellite measurements revealed high horizontal gradients of $D_{m}$ in the vicinity of the hub. This variability was also clearly seen in the radar-radiometer data. For the most part of the cloud, characteristic sizes of cloud particles at 2109 UTC were about 10\%-15\% less than those for 2111 UTC. Doppler radar measurements showed about $20 \mathrm{~m}$ $\mathrm{s}^{-1}$ horizontal winds at cloud altitudes. This corresponds to about $2.5 \mathrm{~km}$ of cloud advection in 2 min between the two profiles shown in Fig. 4.

Two aircraft passes (one from King Air at 2108 UTC and the other from Sabreliner at 2116 UTC) were available approximately at the time of the satellite data. The $D_{m}$ values from the aircraft samples are also shown in Fig. 4 at the corresponding aircraft altitudes. The King Air data point at 2114 UTC is not shown here because it was sampled at the very bottom of the cloud $(6.2 \mathrm{~km})$ where vertical gradients of $D_{m}$ are very large (more than $100 \mu \mathrm{m}$ per $100 \mathrm{~m}$ ). Note that this data point yielded the largest discrepancy in Fig. 2 where the aircraft-derived size exceeded the radar-radiometer-derived size by about a factor of 2 .

For a better comparisons with satellite data, vertically averaged values of $D_{m}$ were calculated for both profiles of particle sizes retrieved from radar-radiometer data. These IWC-weighted average values were $\bar{D}_{m} \approx 140$ $\mu \mathrm{m}$ for 2109 UTC and $\bar{D}_{m} \approx 190 \mu \mathrm{m}$ for $2111 \mathrm{UTC}$, respectively. This is somewhat larger than $\bar{D}_{m} \approx 120$ $\mu \mathrm{m}$ inferred from satellite measurements. Note that, given uncertainties of both approaches, there is quite a good agreement between satellite and radar-radiometer data for 2109 UTC. As was mentioned before, the horizontal separation between 2109 and 2111 UTC in ground-derived data could be as small as $2.5 \mathrm{~km}$, which could be within the uncertainty of the instrumentation hub location in the satellite map presented by Ou et al. (1995). Another possible explanation of a smaller particle size derived from satellite is that satellite vertically averaged data may be somewhat biased toward the cloud top (where particle sizes are generally smaller) for thick clouds.

Another radiatively important cloud parameter derived from satellite and ground-based measurements is cloud optical thickness. As reported by Ou et al. (1995), the satellite-derived visible extinction optical thickness at 2111 UTC is somewhat less than 1. The IR extinction optical thickness derived using ground-based radiometers was about 1.02 for this time. Unfortunately, no independent continuous recordings of cloud optical thickness in the vertical direction were available for this experiment to compare with our radiometer-derived values. The 1995 Arizona Program, however, provided a good opportunity for such comparisons, which are discussed in the next section.

\section{Comparisons of cloud optical thickness obtained from IR radiometer and lidar data}

The optical thickness is one of the essential parameters determining cloud radiative properties. The extinction/absorption $\tau_{\text {e/a }}$ optical depth is defined as

$$
\tau_{\mathrm{e} / \mathrm{a}}=\int_{h_{b}}^{h_{t}} \int_{D_{\min }}^{D_{\max }} Q_{\text {e/a }}(D) A(D) N(D, h) d D d h,
$$

where $Q_{\text {ela }}$ is the extinction/absorption efficiency of a particle with size $D, A$ is the particle cross-sectional area, and the integration is carried out from the height of cloud base $h_{b}$ to the height of cloud top $h_{t}$ (with respect to $h$ ), and from the minimum size $D_{\min }$ to the maximum size $D_{\max }$ (with respect to particle sizes).

The ice particle absorption for visible wavelengths is very small, and almost all the extinction is due to scattering. The particles are large compared to these wavelengths, so $Q_{e}$ is about 2 . At the thermal IR wavelengths $(\lambda \sim 10-12 \mu \mathrm{m})$, absorption is already significant. Here, $Q_{e}$ and $Q_{a}$ are approaching 2 and 1, respectively, when $D$ for solid ice spheres begins to exceed about 25-30 $\mu \mathrm{m}$ (Matrosov et al. 1992). So, if cloud particles are not very small, one can expect $\tau_{e} \approx 2 \tau_{a}$ and $\tau_{e}$ will be approximately the same in the visible and thermal IR regions.

The Arizona Program (January-March 1995) was a multiagency experiment devoted mainly to studies of orographically modified winter storms. However, be- 
tween the storms, some good ice cloud data were collected. The ground-based instrumentation hub of this field experiment was located in Cottonwood, Arizona. The ETL instrumentation included the $\mathrm{K}_{\mathrm{a}}$-band radar and IR and microwave radiometers, as discussed in section 4. The University of Wisconsin operated a groundbased high spectral resolution lidar (HSRL) (Piironen and Eloranta 1994). The ETL radiometers and HSRL were collocated, and their measurements were used to estimate IR and visible cloud optical thicknesses, respectively. These estimations provided data for comparisons discussed below.

\section{a. Retrieval of cloud IR optical thickness from ground-based radiometer measurements}

As mentioned before, obtaining cloud IR optical thickness is a part of the radar-radiometer method for the retrieval of cloud microphysics [see Eq. (1)]. The estimation of the IR absorption optical thickness $\tau_{a}$ from the IR-radiometer brightness temperature $T_{b}$ is based on the equation

$$
\begin{aligned}
B\left(T_{b}\right)= & B\left(T_{e}\right)\left[1-\exp \left(-\tau_{a}\right)+\delta \epsilon\right] P_{a} \\
& +\left(1-P_{a}\right) B\left(T_{a}\right)+R_{g},
\end{aligned}
$$

where $B$ is the Planck function for the middle of the IR-radiometer wavelength band. The correction term $\delta \epsilon$ accounts for the effects of multiple scattering. Here, $T_{e}$ and $T_{a}$ are the cloud and atmosphere effective emitting temperatures, respectively, and $P_{a}$ is the mean atmospheric transmittance in the IR-radiometer wavelength band. The effective emitting temperature of cloud (atmosphere) is defined as the thermodynamic temperature of such an isothermic cloud (atmosphere), which emits the same amount of radiation as the given cloud (atmosphere). Here, $R_{g}$ accounts for the cloud reflection of the ground radiation.

A discrete ordinate algorithm for radiative transfer calculations described by Stamnes et al. (1988) was used to a estimate multiple scattering correction $\delta \epsilon ; \delta \epsilon$ accounts for the contribution of the radiance entering the emergent beam from multiple-scattering processes within the cloud and also for the extinction of the radiation in this beam due to volume scattering. The first effect enhances the brightness temperature of the downwelling radiation, and the second effect reduces it.

The calculations were performed for cirrus models with optical properties discussed by Minnis et al. (1993). This calculation showed that $\delta \epsilon$ was monotonically increasing with cloud optical thickness $\left(\tau_{e}<4\right)$. The maximum values of $\delta \epsilon$ do not exceed about 0.03 for all the considered cirrus models. This constitutes not more than about $4 \%$ of the multiple-scattering contribution to the cloud emissivity calculated under the assumption of the pure absorbing media

$$
\epsilon=1-\exp \left(-\tau_{a}\right)
$$

Negative and positive contributions of multiple scatter-

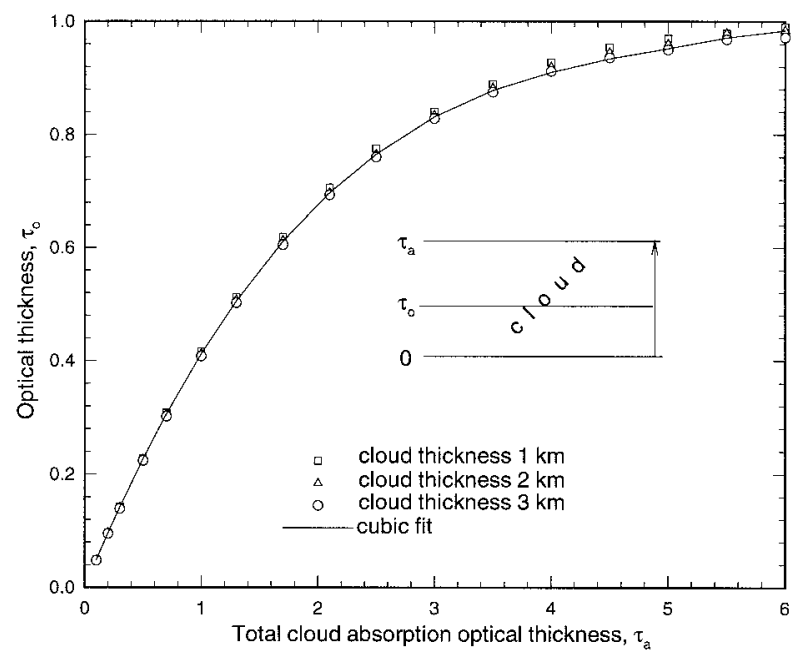

FIG. 5. Optical thickness from the cloud base to a level in a cloud where its thermodynamic temperature is equal to the effective emitting temperature $T_{e}$ of the entire cloud: $B\left(T_{e}\right)=B\left[T\left(\tau_{o}\right)\right](\lambda \sim 10-$ $11.4 \mu \mathrm{m})$.

ing to the brightness temperature of the downwelling radiation effectively cancel each other out and are often neglected.

The atmospheric thermal radiation and transmittance in the IR "window" wavelength region are due mostly to the water vapor continuum. Here, $T_{a}$ is estimated from temperature and humidity profiles known from the radiosonde soundings (or from climatological mean profiles if radiosonde soundings are not available); $T_{a}$ is approximately equal to the thermodynamic temperature at an altitude of about $1 \mathrm{~km}$ above the ground because most of the atmospheric water vapor is concentrated near the surface. Thermal radiation of the atmosphere above and within the cloud is very small compared to that of the atmospheric layer under the cloud and could be neglected.

In an actual cloud, temperature at the cloud bottom and cloud top can differ from a few degrees Celsius to more than $20^{\circ} \mathrm{C}$ depending on the cloud geometrical thickness. Model calculations of cloud IR emission were performed assuming a standard in-cloud vertical temperature gradient of $6.5^{\circ} \mathrm{C} \mathrm{km}^{-1}$ for cloud geometrical thicknesses of 1,2 , and $3 \mathrm{~km}$ and for variable cloud optical thickness $\left(0<\tau_{a}<5\right)$. The results of these calculation are shown in Fig. 5. On the vertical axis in this figure, the optical thickness inside the cloud $\tau_{o}$ is shown where the effective emitting temperature is equal to the cloud thermodynamic temperature.

As seen in Fig. 5, cloud geometrical thickness has a negligible effect on the result, which can be approximated by the cubic polynomial fit in terms of cloud total absorption optical thickness $\tau_{a}$ :

$$
\tau_{o}=\tau_{a}\left(0.006 \tau_{a}^{2}-0.09 \tau_{a}+0.5\right) .
$$

The use of the approximation (8) requires knowing the vertical profile of the cloud absorption coefficient. This 
profile is calculated at each iterative step of the microphysical retrievals using the radar-radiometer method [see Eq. (1)].

The transmittance $P_{a}$ is determined mostly by the total amount of the atmospheric water vapor (WV) available continuously from microwave radiometer measurements. Here, $P_{a}$ can be estimated directly during clearsky measurements or from theoretical calculations for a given radiosonde sounding. Our experience in estimating $P_{a}$ for different experimental situations using these approaches shows that very often the following simple approximation gives satisfactory results (for 0.8 $\mathrm{cm}<\mathrm{WV}<3 \mathrm{~cm})$ :

$$
P_{a} \approx 1.02 \exp (-0.103 \mathrm{WV}) \text {. }
$$

The term $R_{g}$ in (6) is estimated as in Platt and Dilley (1979). They showed that the cloud reflectance depends on optical thickness. This term does not, however, contribute much. For example, for the cloud emissivity of 0.5 , it represents about $5 \%-10 \%$ of the cloud emission, depending on the atmospheric temperature.

\section{b. Retrieval of cloud visible optical thickness from HSRL data}

The University of Wisconsin HSRL measures optical properties of clouds and atmosphere by separating the Doppler-broadened molecular (Rayleigh) backscatter return from the unbroadened aerosol (cloud) particle return. This is possible because the spectral width of light (HSRL operates at $\lambda=532 \mathrm{~nm}$ ) scattered from molecules is broadened due to Doppler shifts caused by the molecular thermal motion. The thermal motion of aerosol and cloud particles is much slower and the backscatter spectrum is nearly unchanged. The molecular backscatter cross section can be calculated from the molecular density profile. The molecular profile is then used as a calibration target that is available at each point in the lidar return signal profile. This calibration allows unambiguous measurements of aerosol (cloud) extinction profile by comparing the magnitude of the measured molecular return signal relative to the computed signal. This procedure is described by Piironen and Eloranta (1994) in more detail.

A very small field of view of the receiving telescope (160 $\mu \mathrm{rad})$ minimizes multiple scattering contributions to the received signals. Visible extinction optical thickness is calculated as the vertical integral of the extinction coefficient. The lidar measurements of cloud properties are available up to $\tau_{e} \approx 2.5$. For larger optical thicknesses, severe round-trip attenuation prevents obtaining any cloud information from these measurements.

Lidar measurements of cloud optical thickness are more robust than the radiometric measurements because they make fewer model assumptions. However, there are some advantages of radiometric measurements too. One of the most important ones is that the radiometric measurements allow estimating optical thickness up to $\tau_{a} \approx 2.5-3$ before the radiation saturation effects prevent such estimations. This increases the cloud optical thickness limit by about a factor of 2 compared to lidar measurements because in the infrared $\tau_{e} \approx 2 \tau_{a}$.

\section{c. Comparisons of retrieved IR and visible optical thicknesses}

Figure 6 shows comparisons of optical thickness values retrieved from IR radiometric and visible lidar measurements for four cases of high-altitude ice clouds. For a better comparison, IR results, as visible data, are given in terms of extinction optical thickness $\tau_{e}$. There are important sampling differences between radiometer and lidar measurements that should be kept in mind when analyzing the optical thickness data. The most important difference is in the angular resolution, which is much finer for HSRL $(\sim 160 \mu \mathrm{rad})$ than for IR radiometer $\left(\sim 2^{\circ}\right)$. Infrared measurements also imply shorter averaging time (30 s) compared to 3-min averaging for lidar data. For easier comparisons, lidar and radiometer data are shown for the centers of time intervals during which they were collected.

The angular resolution difference is of lesser importance for more horizontally homogeneous cases when optical thickness changes relatively slowly. Such cases are represented in Figs. 6a and 6c. For these cases, the cloud base was between about 7 and $8 \mathrm{~km}$ and geometrical thickness was changing from a few hundred meters up to about $3 \mathrm{~km}$ over the observation time. Comparisons of $\tau_{e}$ for these clouds show a good agreement. The relative standard deviations of visible and IR optical thicknesses are about $17 \%$ for both cases.

Unlike the discussed cases of 30 January (Fig. 6a) and 18 February (Fig. 6c), the 7 February case (Fig. 6b) represents a very inhomogeneous "patchy" cloud with abrupt changes of cloud optical thickness. For a significant period of the observation time, $\tau_{e}$ was greater than 2.5, which prevented lidar measurements (corresponding data are shown as a straight horizontal line at $\left.\tau_{e}=2.5\right)$. The cloud optical thickness for most of this period was between 2.5 and 6 as obtained from IR radiometer data that were not yet saturated. Note that two 3-min data points of lidar data during this period (about 2054 UTC and 2138 UTC) yielded $\tau_{e}$ between 1 and 2 with IR data showing much higher values. The most probable reason for this discrepancy is that lidar was looking in between cloud regions with large $\tau_{e}$ that dominated the much larger field of view of the IR radiometer. The agreement between IR and visible data is quite good in the beginning and the end of the observation time when the cloud optical thickness was less than 2 .

The case of 3 March, shown in Fig. 6d, is intermediate between the case of 7 February and the cases of 30 January and 18 February. The cloud optical thickness was changing significantly from 1 to about 3 . These changes were, however, not as abrupt as during the 7 February case, and both instruments provided contin- 

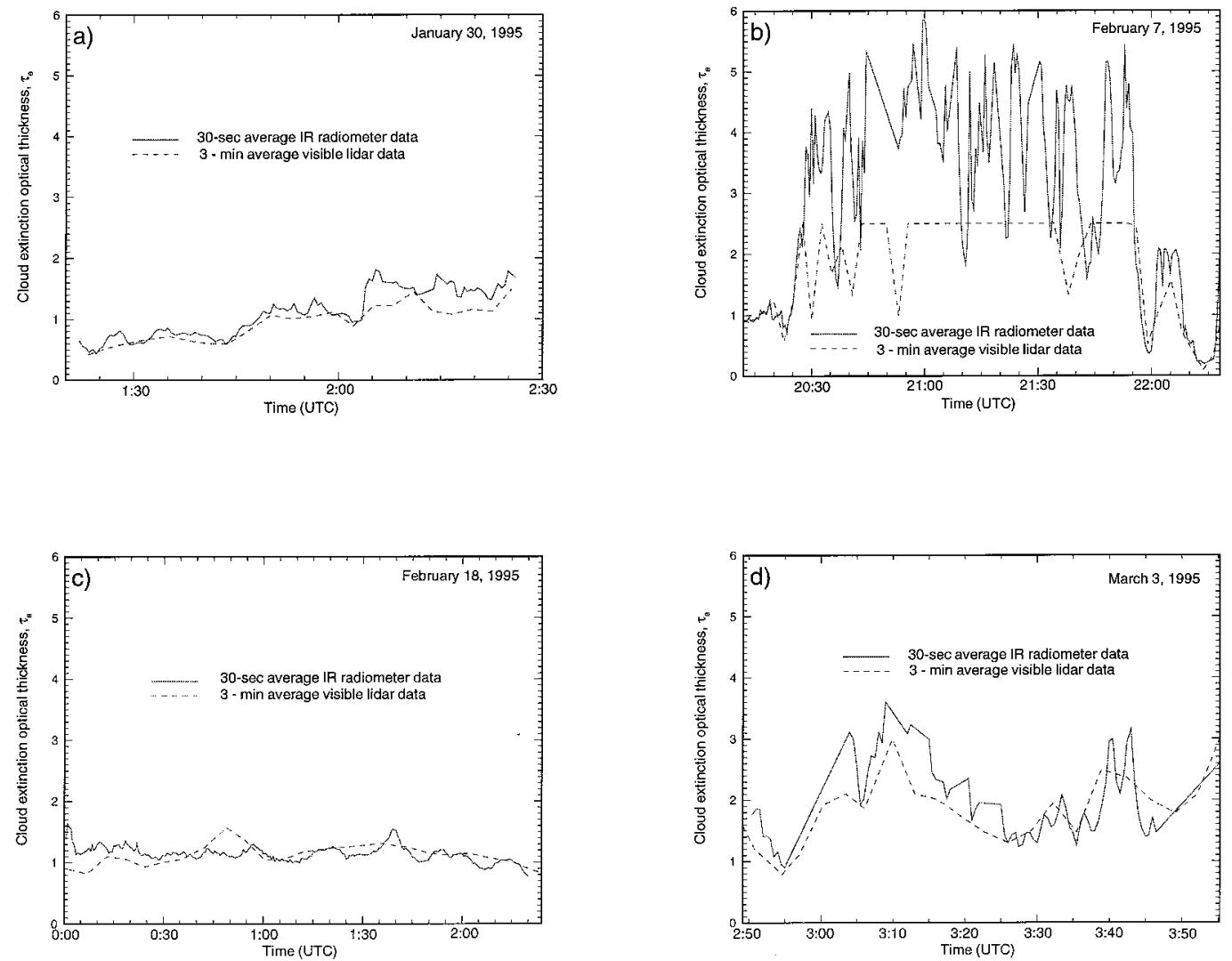

FIG. 6. Comparisons of ice cloud extinction optical thickness derived from the ground-based visible lidar and IR radiometer measurements during the 1995 Arizona Program.

uous datasets. The agreement between IR and visible $\tau_{e}$ for this case is satisfactory-the standard deviation between the two is about $20 \%$.

None of the considered optical thickness comparisons revealed a systematic excess of visible extinction compared to IR extinction. Such excess could be expected when cloud particles are large compared to visible wavelengths but not large compared to the IR "window" wavelengths. The observed result indicates that, at least in the considered clouds, the contribution to the total IR extinction from small particles (less than about 20 $\mu \mathrm{m})$ was not significant compared to larger particles with extinction efficiencies close to 2 .

\section{Conclusions}

Comparisons of the ice cloud microphysical parameters retrieved using the ground-based radar-radiometer remote sensing method and direct in situ aircraft sampling were performed for the FIRE-II experimental case of 26 November 1991. Because of the high temporal and spacial variability of cloud parameters, quantitative analysis was performed only for data when the aircraft were sampling clouds above the ground-based, vertically pointed radar and radiometer. This ensured at least nearly the same regions of the clouds were sampled directly and remotely.

The remotely and directly obtained characteristic particle sizes expressed in median mass diameters of equalvolume spheres agreed with the relative standard deviation of about $33 \%$ between them. This agreement was approximately the same for the King Air 2D data, which included data for particles greater than about 18 $\mu \mathrm{m}$, and for the Sabreliner combined 2D and video analysis data, which accounted for a very large dynamic range of particle sizes from about $5 \mu \mathrm{m}$ to several millimeters.

The relative standard deviation between IWC values measured by aircraft and those retrieved from radarradiometer data was $55 \%$. This result should be considered very encouraging given uncertainties of both direct and remote approaches and the very high variability of IWC. This variability exceeds four orders of magnitude for natural clouds and was more than two orders of magnitude for data sampled here. Again, as for characteristic particle sizes, there were no significant differences between agreement of remote data with both types of aircraft data, which indicates the low contribution of small particles (less than about $25 \mu \mathrm{m}$ ) total cloud IWC for the considered data set. 
A demonstrated good agreement between the remotely derived ice cloud microphysical parameters and established techniques of aircraft in situ sampling gives a certain confidence in the results of the radar-radiometer method. However, more extensive comparisons of such kind are still needed to fully understand potentials of the remote method in different cloud conditions.

The comparisons of reflectivity $Z_{e}$ measured by the radar and calculated from aircraft data using particle mass-size relationships revealed that calculated values were about $2 \mathrm{~dB}$ lower than those measured with the radar. Such discrepancy is, however, well within the uncertainty of using different existing mass-size relationships for cirrus particles and measurement errors. This small discrepancy also has contributions from particle nonsphericity, which were neglected when calculating $Z_{e}$ from particle samples and also from radar calibration errors.

Ground-based, radar-radiometer remote measurements of cloud particle characteristic size were also compared to the satellite-derived size available for the considered period of comparisons between remote and aircraft direct data for 26 November 1991. The satellite data expressed in the median mass diameter of the equalvolume sphere provided a vertically averaged mean value of about $120 \mu \mathrm{m}$, while the corresponding mean data for the vertical size profiles from the ground-based remote sensors gave 140-190 $\mu \mathrm{m}$ for the profiles retrieved within 2 min from the time of satellite measurements. The vertical variability of median particle sizes for these profiles was very large: from about $50 \mu \mathrm{m}$ to more than $200 \mu \mathrm{m}$, with larger particles in the lower part of the cloud, which was about $3.5 \mathrm{~km}$ thick at this time.

The 1995 Arizona Program field experiment provided opportunities to compare visible and IR cloud optical thickness derived from lidar and thermal IR radiometer measurements, respectively. Such comparisons for the cases with no abrupt changes in cloud optical thickness showed that visible and IR extinction optical thicknesses usually differed from each other by not more than $20 \%$ (as expressed in terms of the relative standard deviation). No systematic excess of visible extinction compared to IR extinction was observed.

The remote sensing methods for clouds are still quite new. More comparisons between different retrieval methods and in situ sampling should be made to better establish the reliability and quality of these methods and the range of the appropriate conditions in which they can be applied. In this article, we summarize the currently available data that are suitable for testing the radar-radiometer method. These early results are encouraging and offer promise for providing continuous estimation of cloud microphysical properties for climate modeling applications.

The combination of active (radar) and passive (radiometer) data makes remote sensing methods potentially more powerful than passive methods alone, such as those currently relied on by satellites. Of course, satellites offer the only means of obtaining global coverage of cloud conditions. Thus, the most promising application of the ground-based method may serve as a means for "tuning" of the satellite measurements over selected "calibration/validation" sites. Additional evaluations of the different versions of the radar-radiometer method with in situ direct data from aircraft of balloon and the use of the remotely derived microphysical information in climate models are priorities of the Atmospheric Radiation Measurement (ARM) program (Stokes and Schwartz 1994), which operates the appropriate remote sensors at a few ground-based sites around the world. Further advances of the remote sensing methods are expected from ARM and related projects.

Acknowledgments. This work was supported by the Environmental Science Division of the U.S. Department of Energy as part of the Atmospheric Radiation Measurement Program and the NOAA Office of Global Programs. Experimental data were collected during field programs funded by NASA (FIRE-II) and NOAA and Arizona Department of Water Resources (1995 Arizona Program).

\section{REFERENCES}

Atlas, D., M. Kerker, and W. Hitschfeld, 1953: Scattering and attenuation by non-spherical atmospheric particles. J. Atmos. Terr. Phys., 3, 108-119.

Brown, P. R. A., and P. N. Francis, 1995: Improved measurements of ice water content in cirrus using a total-water probe. J. Atmos. Oceanic Technol., 12, 410-414.

—, A. J. Illingworth, A. J. Heymsfield, G. M. McFarquhar, K. A. Browning, and M. Gosset, 1995: The role of spaceborne millimeter-wave radar in the global monitoring of ice cloud. J. Appl. Meteor., 34, 2346-2366.

Dowling, D. R., and L. F. Radke, 1990: A summary of the physical properties of cirrus clouds. J. Appl. Meteor., 29, 970-987.

Heymsfield, A. J., 1977: Precipitation development in stratiform ice clouds: A microphysical and dynamical study. J. Atmos. Sci., 34, 367-381.

, and G. M. McFarquhar, 1996: High albedos of cirrus in the tropical Pacific warm pool: Microphysical interpretations from CEPEX and from Kwajalein, Marshall Islands. J. Atmos. Sci., 53, 2424-2450.

_, K. M. Miller, and J. D. Spinhirne, 1990: The 27-28 October 1986 FIRE IFO cirrus case study: Cloud microstructure. Mon. Wea. Rev., 118, 2313-2328.

Klimowski, B. A., 1995: The 1995 Arizona Program. Field Operation Plan, The University of Arizona, $67 \mathrm{pp}$. [Available from Department of Atmospheric Sciences, The University of Arizona, Tucson, AZ 85721.]

Kosarev, A. L., and I. P. Mazin, 1989: Empirical model of physical structure of the upper level clouds of the middle latitude. Radiation Properties of Cirrus Clouds, E. M. Feigelson, Ed., Nau$\mathrm{ka}, 29-52$.

Liao, L., and K. Sassen, 1994: Investigation of relationships between Ka-band radar reflectivity and ice and liquid water content. Atmos. Res., 34, 231-248.

Matrosov, S. Y., 1993: Possibilities of cirrus particle sizing from dualfrequency radar measurements. J. Geophys. Res., 98, $20675-$ 20683.

, 1997: Variability of microphysical parameters in high-altitude ice clouds: Results of the remote sensing method. J. Appl. Meteor., 36, 633-648. 
T. Uttal, J. B. Snider, and R. A. Kropfli, 1992: Estimation of ice cloud parameters from ground-based infrared radiometer and radar measurements. J. Geophys. Res., 97, 1567-1574.

— B. B. Orr, R. A. Kropfli, and J. B. Snider, 1994: Retrieval of vertical profiles of cirrus cloud microphysical parameters from Doppler radar and infrared radiometer measurements. J. Appl. Meteor., 33, 617-626.

— A. J. Heymsfield, J. M. Intrieri, B. W. Orr, and J. B. Snider 1995: Ground-based remote sensing of cloud particle sizes during the 26 November 1991 FIRE-II cirrus case: Comparisons with in situ data. J. Atmos. Sci., 52, 4128-4142.

McFarquhar, G. M., and A. J. Heymsfield, 1996: Microphysical characteristics of three anvils sampled during the Central Equatorial Pacific experiment. J. Atmos. Sci., 53, 2401-2423.

Minnis, P., K. N. Liou, and Y. Takano, 1993: Inference of cirrus cloud properties using satellite-observed visible and infrared radiances. Part I: Parameterization of radiance fields. J. Atmos. Sci., 50, 1279-1304.

Mitchell, D. L., 1996: Use of mass- and area-dimensional power laws for determining precipitation particle terminal velocities. J. Atmos. Sci., 53, 1710-1723.

Ou, S. C., and Coauthors, 1995: Remote sounding of cirrus cloud optical depth and ice crystal sizes from AVHHR data: Verification using FIRE II IFO measurements. J. Atmos. Sci., 52, $4143-4158$.
Piironen, P., and E. W. Eloranta, 1994: Demonstration of a high spectral resolution lidar based on an iodine absorption filter. Opt. Lett., 19, 234-236.

Platt, C. M. R., and A. C. Dilley, 1979: Remote sensing of high clouds. Part II: Emissivity of cirrostratus. J. Appl. Meteor., 18, 1144-1150.

Randall, D. A., 1995: Atlantic Stratocumulus Transition Experiment. J. Atmos. Sci., 52, 2705-2706.

Sassen, K., 1987: Ice cloud content from radar reflectivity. J. Climate Appl. Meteor., 26, 1050-1053.

_ 1990 : Is there a cirrus small particle radiative anomaly? Preprints, Conf. on Cloud Physics, San Francisco, CA, Amer. Meteor. Soc., J91-J95.

Stamnes, K., S-C. Tsay, W. Wiscombe, and K. Jayaweera, 1988: Numerically stable algorithm for discrete-ordinate method radiative transfer in multiple scattering and emitting layered media. Appl. Opt., 27, 2502-2509.

Starr, D. O'C., and D. P. Wylie, 1990: The 27-28 October 1986 FIRE cirrus case study: Meteorology and clouds. Mon. Wea. Rev., 118, 2259-2287.

Stephens, G. L., 1995: First ISCCP Regional Experiment Intensive Field Operations II. J. Atmos. Sci., 52, 4041-4042.

Stokes, G. M., and S. E. Schwartz, 1994: The atmospheric radiation measurement (ARM) program: Programmatic background and design of the cloud and radiation test bed. Bull. Amer. Meteor. Soc., 75, 1201-1221. 